\title{
OPENNESS, TECHNOLOGICAL SPILLOVERS IN THE R\&D SECTOR AND ECONOMIC GROWTH
}

\author{
Pei-Pei Chen and Rangan Gupta \\ Department of Economics, University of Pretoria
}

Accepted June 2009

\begin{abstract}
Recent studies have pointed out that trade liberalisation leads to technological spillovers, which tend to improve the efficiency of the domestic research and development (R\&D) sector, and ultimately boost economic growth. In this paper, we theoretically formalise the above-mentioned relationship between trade openness and growth, via knowledge spillover in the R\&D sector. We show that, under certain conditions, an increase in the degree of openness not only enhances growth, but also improves the standard of living. The study, thus, prescribes policies of developing and improving the domestic R\&D sector in order to reap the benefits of trade liberalisation.
\end{abstract}

Keywords: Trade openness; growth; research and development.

JEL F43; O32; 41

\section{1 Introduction}

The importance of the relationship between trade and economic growth has been realised ever since Adam Smith's and David Ricardo's theories of absolute and comparative advantages, respectively. Over the years, empirical research ${ }^{1}$, in general, ${ }^{2}$ has vindicated a strong and positive correlation between openness and economic growth, after controlling for other explanatory variables explaining economic growth. The result is robust across methodologies, model specifications, sample of countries and time frames. Openness can affect growth for various reasons,${ }^{3}$ but, recent studies by Coe and Helpman (1995), Branstetter (1996), Keller (1998) and Nadiri and Kim (1996) have singled out the role of technological spillovers, resulting from trade liberalisation, that tend to improve the efficiency of the domestic research and development (R\&D) sector, which ultimately boosts economic growth. ${ }^{4}$

This paper theoretically formalises the abovementioned relationship between trade openness and growth via technological and knowledge spillover in the R\&D sector. We extend the R\&D model of Romer (2006) by incorporating the role of openness and its impact on the domestic technological sector. We show that, under certain conditions, openness is growth enhancing and also improves the standard of living. The study, thus, stresses on the importance of policies directed towards developing and improving the domestic R\&D sector in order to reap the full benefits of trade liberalisation. The remainder of the paper is organised as follows: besides the introduction and conclusions, section 2 presents the theoretical model, while section 3 solves and discusses the basic results of the modified R\&D model, following an increased degree of openness.

\section{2}

\section{Research and development framework}

The theoretical model is based on Romer's (2006) Research and Development (R\&D) Model. Essentially, it makes an attempt 
to endogenise the technological progress, which has otherwise generally been treated as exogenous in the standard Solow-type models. The model involves four variables: labour $(L)$, capital $(K)$, technology $(A)$ and output $(Y)$. There are two sectors: a goods-producing sector (equation 1) where outputs are produced and a R\&D sector (equation 2) where knowledge is accumulated. Fraction $a_{l}$ of the labour force is used in the R\&D sector and fraction $1-a_{l}$ is used in the goods-producing sector. Similarly $a_{k}$ of the capital stock is used in the R\&D sector and the rest in goods production. Both $a_{l}$ and $a_{k}$ are exogenous and constant. Both sectors use the full stock of knowledge $A$, since the usage of an idea does not prevent it from being used elsewhere.

The quantity of output produced at time $t$ is given by:

$$
\begin{aligned}
& Y(t)=\left[\left(1-a_{k}\right) K(t)\right]^{\alpha}\left[A(t)\left(1-a_{l}\right) L(t)\right]^{1-\alpha}, \\
& 0<\alpha<1
\end{aligned}
$$

Note $\alpha(1-\alpha)$ is the output elasticity of capital (effective labour) used in the output sector. Equation (1) is a standard constant return to scale Cobb-Douglas production function in capital and labour. As shown in equation (2), the quantity of knowledge is produced via a generalised Cobb-Douglas type production function.

$$
\begin{aligned}
& \dot{A}=B\left[a_{k} K(t)\right]^{\beta}\left[a_{l} L(t)\right]^{\gamma}[A(t)]^{\theta}, \\
& B>0, \beta \geq 0, \gamma \geq 0,
\end{aligned}
$$

where $B$ is a shift parameter and $\beta$ and $\gamma$ are, both the elasticity of new knowledge creation with respect to capital and labour.

Unlike in the output sector, the production function for knowledge is not assumed to have constant returns to scale for labour and capital. As Romer (2006) points out, the standard argument that there should be at least constant returns, is based on a replication issue with the new inputs doing exactly what the old inputs were doing, if we double the input, we double the output. But in the case of the knowledge production sector, exactly replicating what the existing inputs were doing would merely result in the same set of discoveries to be made twice, and, hence, keep $\dot{A}$ unchanged. Thus, it is possible to have diminishing returns in the R\&D sector. At the same time, fixed costs of setup, interaction among researchers and so on, may be important enough in the R\&D sector to cause the doubling of capital and labour to more than double output. We thus also allow for the possibility of increasing returns. Moreover, there is no reason as to how we should restrict the increases in the existing stock of knowledge to affect the production of new knowledge, and hence, we place no restriction on $\theta$ to start off. $^{5}$

Our departure from Romer's (2006) model lies in our treatment of $\theta$. In our case $\theta$ is not exogenous, but a positive function of the degree of openness of the economy in concern. Such an assumption enables us to account for the advanced informational and technological spillovers that result from opening up to the global economy. However, the percentage change in the accumulation of new knowledge via an increase in openness with respect to a one percentage change in the existing stock of knowledge, increases at a decreasing rate. This implies that with unchanged capital and labour, openness causing better utilisation of the existing knowledge base, through spillovers, will have diminishing returns on new knowledge creation after a certain stage. Formally, we choose the following specification for $\theta$ :

$\theta=\underline{\theta}+c \lambda^{\vartheta}, \underline{\theta}>0, c>0, \lambda>0,0<\vartheta<1$

Note such a formulation implies that, as the degree of openness $(\lambda)$ intensifies, one percentage increase in the current stock of knowledge, given capital and labour, would produce a higher percentage increase in the creation of new knowledge. This is purely a result of better know-how of utilising the existing stock of knowledge resulting from the information spillovers following trade liberalisation. However, the increase occurs at a decreasing rate, since $0<\vartheta<1$. Moreover, when the economy is closed, that is $\lambda=0, \theta$ is at its lower limit of $\theta$. Finally, note that $c$ is a positive scalar which is chosen in a manner to ensure that equilibrium exists. ${ }^{6}$

Like in the Solow model, savings is equivalent to investments: $S=I$, with the saving rate, $0<s<1$, being exogenous and constant. Thus, 
with the depreciation rate set equal to zero for simplicity, we have:

$\dot{K}=s Y(t)$

The population grows at an exogenous rate of $n$, which implies:

$\frac{\dot{L}}{L}=n$

There are two endogenous stock variables, $A$ and $K$. Substituting the production function (1) into the expression for capital accumulation yields

$\dot{K}=s\left[\left(1-a_{k}\right) K(t)\right]^{\alpha}\left[A(t)\left(1-a_{l}\right) L(t)\right]^{1-\alpha}$

$$
\begin{aligned}
& \ln g_{k}=\ln c_{k}+(1-\alpha)[\ln A(t)+\ln L(t)-\ln K(t)] \\
& \frac{\dot{g}_{k}}{g_{k}}=(1-\alpha)\left[g_{A}+n-g_{k}\right]
\end{aligned}
$$

where $g_{A}=\frac{\dot{A}}{A}$. Note, from (10) $g_{k}$ is rising if $\left[g_{A}+n-g_{k}\right]$ is positive, and falling if this expression is negative, and constant if it is zero. The information is summarised in Figure 1. In the $\left(g_{A}, g_{k}\right)$ plane, the locus of points where $g_{k}$ is constant has an intercept of $n$ and a slope of 1 .
Dividing both sides by $K$ and defining $c_{k}=$ $s\left[\left(1-a_{l}\right)^{1-\alpha}\left(1-a_{k}\right)^{\alpha}\right]$ gives an expression of

$$
\begin{aligned}
g_{k} & =\frac{\dot{K}}{K} \\
& =s\left[\frac{A(t) L(t)}{K(t)}\right]^{1-\alpha}\left[\left(1-a_{l}\right)^{1-\alpha}\left(1-a_{k}\right)^{\alpha}\right] \\
& =c_{k}\left[\frac{A(t) L(t)}{K(t)}\right]^{1-\alpha}
\end{aligned}
$$

Taking logs of both sides of (8) and differentiating the same with respect to time yields, simultaneously:

Since from (10) we have:

$g_{k}=n+g_{A}$

Moreover, starting from $\frac{\dot{g}_{k}}{g_{k}}=0$, an increase (decrease) in $g_{A}$ makes causes $g_{k}$ to be positive (negative). Hence, above (below) the $\frac{g_{k}}{g_{k}}=0$ locus, $g_{k}$ is falling (rising).

\section{Figure 1}

The dynamics of the growth rate of capital in the R\&D model

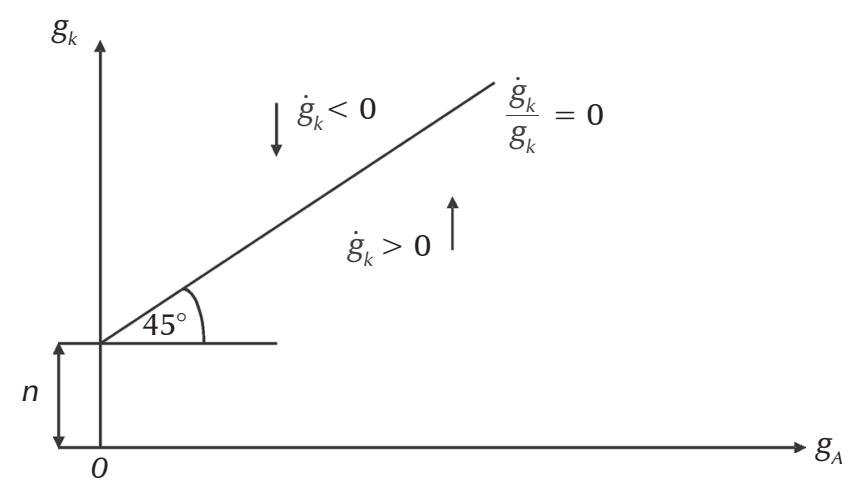

Taking equation (2) and dividing both sides by where $c_{A}=B a_{k}^{\beta} a_{l}^{\gamma}$.

$A$, yields

$$
\begin{aligned}
\frac{\dot{A}}{A}=g_{A} & =B\left[a_{k} K(t)\right]^{\beta}\left[a_{l} L(t)\right]^{\gamma}[A(t)]^{\theta-1} \\
& =c_{A} K(t)^{\beta} L(t)^{\gamma} A(t)^{\theta-1}
\end{aligned}
$$

As in the case of $g_{k}$, taking logs and the time derivative of (13) simultaneously, yields:

$\ln g_{A}=\ln c_{A}+\beta \ln K(t)+\gamma \ln L(t)+(\theta-1) \ln A(t)$ 
$\frac{\dot{g}_{A}}{g_{A}}=\beta g_{k}+\gamma n+(\theta-1) g_{A}$

Note that $\frac{\dot{g}_{A}}{g_{A}}=0$ in the $\left(g_{A}, g_{k}\right)$ plane implies that

$\beta g_{k}=-\gamma n+(1-\theta) g_{A}$

Or, alternatively,

$g_{k}=-\frac{\gamma n}{\beta}+\left(\frac{1-\theta}{\beta}\right) g_{A}$

Firstly, in order to ensure that an equilibrium exists, which simply, requires that $\frac{\dot{g}_{k}}{g_{k}}=0$, that is the two curves of $\frac{\dot{g}_{A}}{g_{A}}=0$ and $\frac{\dot{g}_{A}}{g_{A}}=0$ intersect, the curve must be positively sloped, which, in turn, requires a necessary condition of $\theta<1$. Secondly, the sufficiency condition requires, in addition, that the $\frac{\dot{g}_{A}}{g_{A}}=0$ locus must have a steeper slope than the $\frac{g_{k}}{\alpha}=0$ curve, which implies that $\left(\frac{1-\theta}{\beta}\right)>1$ or $\beta^{\beta}+\theta<1$. And given the specification of $\theta$ in (3), we require $c$ to be constrained by the following condition, given, $\beta, \underline{\theta}, \lambda$ and $\vartheta$ :

$0<c<\frac{1-\beta-\underline{\theta}}{\lambda^{\vartheta}}$

Now given that $\theta<1$, the set of points where $g_{A}$ is constant, that is $\frac{\dot{g}_{A}}{g_{A}}=0$, has an intercept of $-\gamma_{n} / \beta$ and a slope of $\left(\frac{1-\theta}{\dot{g}_{A} \beta}\right)$, is depicted in Figure 2. Note above the $\frac{g_{A} \beta}{g_{A}}=0, g_{A}$ is rising, while it is falling below the locus. This is easy to see since starting from $\frac{\dot{g}_{A}}{g_{A}}=0$, an increase (decrease) in $g_{k}$ makes $g_{A}>0(<0)$.

Figure 2

The dynamics of the growth rate of knowledge in the R\&D model

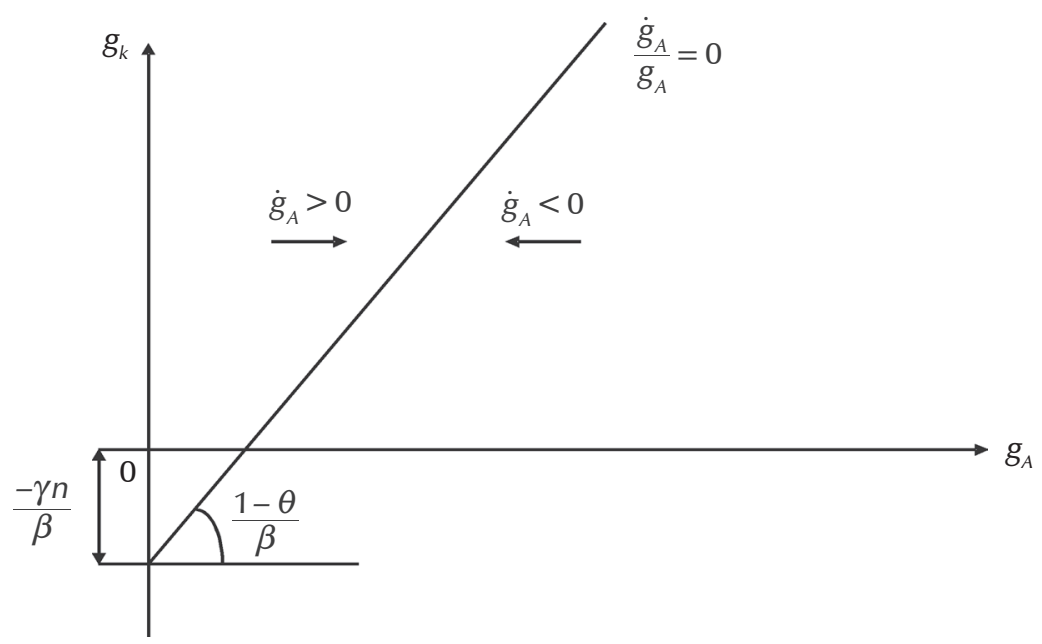

As stated above for the steady-state equilibrium we require: $\frac{\dot{g}_{k}}{g_{k}}=0=\frac{\dot{g}_{A}}{g_{A}}$. Given that (17) holds, we have a unique equilibrium at point E. Moreover, given the behavior of $g_{k}$ and $g_{A}$ below and above the locii: $\frac{\dot{g}_{k}}{g_{k}}=0$ and $\frac{\dot{g}_{A}}{g_{A}}=0$, the equilibrium at $\mathrm{E}$ is also a stable one. ${ }^{7}$ The information is summarised in Figure 3.
Note the corresponding equilibrium values of $g_{k}$ and $g_{A}$ at the equilibrium is denoted by $\hat{g}_{k}$ and $\hat{g}_{A}$ respectively. The values of $\hat{g}_{k}$ and $\hat{g}_{A}$, in turn ensure that at point E, equations (11) and (17) must hold simultaneously. 


\section{Figure 3}

The dynamics of growth rates of capital and knowledge in equilibrium

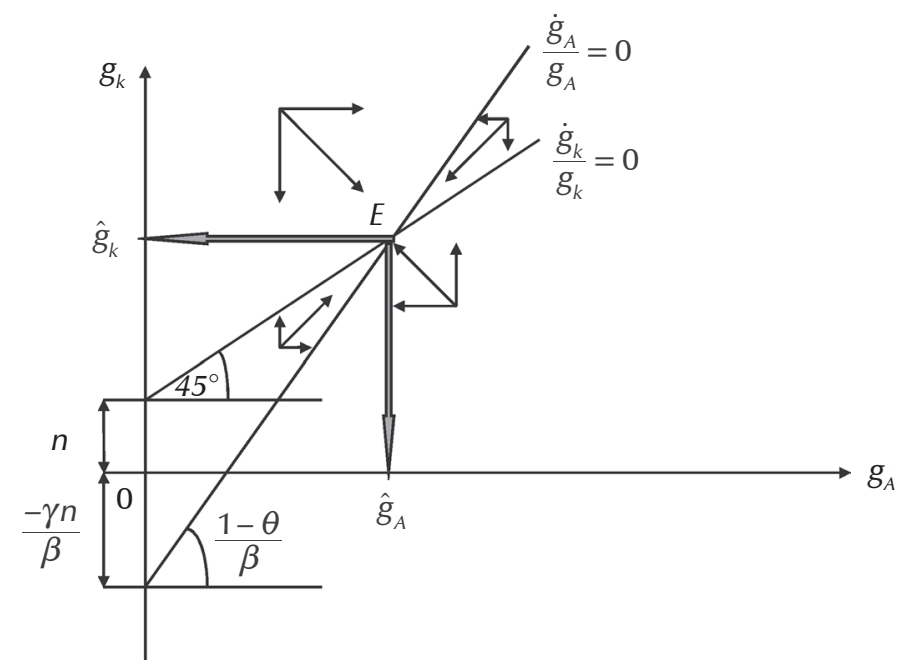

Therefore, at the equilibrium point $\mathrm{E}$, equations (11) and (17) become

$\hat{g}_{k}=\hat{g}_{A}+n$

$\hat{g}_{k}=-\frac{\gamma n}{\beta}+\left(\frac{1-\theta}{\beta}\right) \hat{g}_{A}$

Substituting (19) into (20) yields

$\hat{g}_{A}+n=-\frac{\gamma n}{\beta}+\left(\frac{1-\theta}{\beta}\right) \hat{g}_{A}$

Collecting like terms and simplifying

$\hat{g}_{A}\left\{1-\left(\frac{1-\theta}{\beta}\right)\right\}=-\frac{\gamma n}{\beta}-n$

$\hat{g}_{A}\{\beta+\theta-1\}=-n(\gamma+\beta)$

$\hat{g}_{A}=\frac{n(\gamma+\beta)}{1-(\beta+\theta)}$

And when equation (22) is substituted into (19)

$\hat{g}_{k}=\frac{n(\gamma+\beta)}{1-(\beta+\theta)}+n$

The equation is further simplified

$\hat{g}_{k}=\frac{n \gamma+n \beta+n-n \beta-n \theta}{1-(\beta+\theta)}$

$\hat{g}_{k}=\frac{n+n(\gamma-\theta)}{1-(\beta+\theta)}=\frac{n[1+(\gamma-\theta)]}{1-(\beta+\theta)}$
Moreover, taking logs and the time derivative of the production function for output, equation (1), we obtain the growth rate of output as follows:

$\frac{\dot{Y}}{Y}=g_{y}=\alpha g_{k}+(1-\alpha)\left\{g_{A}+n\right\}$

And, on substituting equation (11) into (25) we have:

$\frac{\dot{Y}}{Y}=\hat{g}_{y}=\alpha \hat{g}_{k}+(1-\alpha) \hat{g}_{k}=\hat{g}_{k}$

$\frac{\dot{Y}}{Y}=\hat{g}_{y}=\hat{g}_{k}$

Finally, we can obtain the growth rate for the standard of living or income per capita, $y=\frac{Y}{L}$, by subtracting the growth rate of labour from the growth rate of output. Using equations (11), (26) and (22), we have the following expression:

$$
\begin{aligned}
& \frac{\dot{y}}{y}=\hat{g}_{y l}=\frac{\dot{Y}}{Y}-\frac{\dot{L}}{L}=\hat{g}_{k}-n=\hat{g}_{A} \\
& =\frac{\beta+\gamma}{1-(\theta+\beta)} n
\end{aligned}
$$


3

\section{The effect of openness on economic growth}

After having characterised the equilibrium in section 2, we are now ready to analyse the impact of an increase in the degree of openness $(\lambda)$ on the steady-state growth rate $\left(\hat{g}_{y}\right)$ and the steady-state standard of living $\left(\hat{g}_{y l}\right)$. Given equation (3), that is $\theta=\underline{\theta}+c \lambda \vartheta$, as the degree of openness intensifies, the elasticity of the creation of new knowledge with respect to the existing stock of knowledge $(\theta)$ increases. Given equation (22) and the fact that a steadystate equilibrium exists $\left(0<c<\frac{1-\beta-\underline{\theta}}{\lambda^{\vartheta}}\right)$, an increase in $\theta$ causes the steady-state growth rate of knowledge $\left(\hat{g}_{A}\right)$ to increase. With $\hat{g}_{k}=\hat{g}_{A}+n$, the steady-state growth rate of physical capital $\left(\hat{g}_{k}\right)$ rises following an increase in $\hat{g}_{A}$ and given $n$. The increase in $\hat{g}_{k}$ also implies an increase in the growth rate of output since $\hat{g}_{y}=\hat{g}_{k}$. Finally, given that $\hat{g}_{y l}=\hat{g}_{A}$, an increase in the degree of openness improves the steady-state growth rate of the standard of living of the economy as well.

Figure 4 summarises the effect of the intensification of the degree of openness on the steadystate growth rate of capital and knowledge, given that a unique and stable equilibrium exists. Starting from an initial equilibrium at $E$, an increase in $\theta$ to $\theta_{1}$ due to a rise in the measure of openness from $\lambda$ to $\lambda_{1}$, causes the $\frac{\dot{g}_{A}}{g_{A}}=0$ locus to swing to the right, due to a fall in the slope of the curve. Note $\left(\frac{1-\theta_{1}}{\beta}\right)<\left(\frac{1-\theta}{\beta}\right)$. As a result of this movement the new equilibrium of the economy moves to $E_{1}$ causing the steadystate levels of the growth rate of capital and knowledge, respectively, to rise to $\hat{g}_{k 1}$ and $\hat{g}_{A 1}$ from their initial corresponding values of $\hat{g}_{k}$ and $\hat{g}_{A}$.

\section{Figure 4}

Effect of an increase in the degree of openness on the steady-state growth rate of capital and knowledge

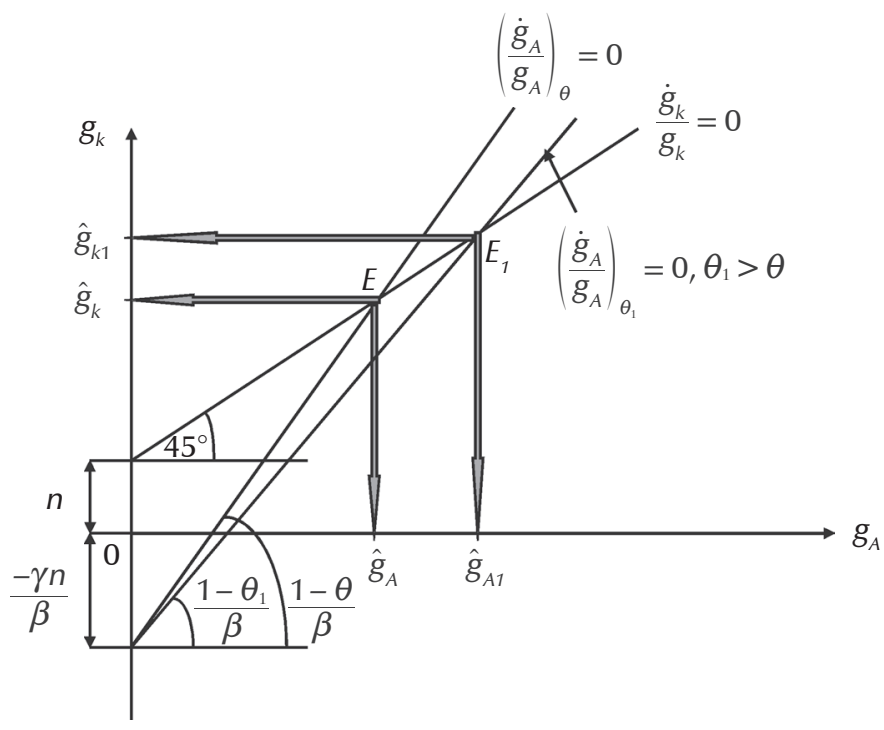

4

\section{Conclusions}

Recent studies by Coe and Helpman (1995), Branstetter (1996), Keller (1998) and Nadiri and Kim (1996) have pointed out that trade liberalisation leads to technological spillovers, which, in turn, tends to improve the efficiency of the domestic research and development (R\&D) sector and ultimately boosts economic growth. 
In this paper, we theoretically formalise the above-mentioned relationship between trade openness and growth via technological and knowledge spillover in the $R \& D$ sector. We show that, under certain conditions, specifically, when the elasticity of new knowledge creation with respect to the existing stock of knowledge is less than one, the sum of the elasticities of new knowledge creation with respect to capital and the existing stock of knowledge is also less than one, an increase in the degree of openness is not only growth enhancing, but also improves the standard of living. The study thus prescribes policies of developing and improving the domestic $\mathrm{R} \& \mathrm{D}$ sector in order to reap the full benefits of trade liberalisation.

\section{Acknowledgement}

We are grateful to two anonymous referees for their comments that helped improve the quality of the revised version.

\section{Endnotes}

1 For a detailed literature review on the theoretical and empirical relationship between openness and growth, see Chen and Gupta (2009).

2 Rodriguez and Rodrik (2000), however, find little evidence that open trade policies are significantly associated with economic growth. They argue that in many cases the indicators of openness used are poor measures of trade barriers or are highly correlated with other sources of bad economic performance. Thus, methodological problems with the empirical strategies employed leave the results of the positive effect of openness on growth open to alternative interpretations.

3 See Aghion and Howitt (1996) for further details.

4 In an interesting study dealing with declining publication rate in South Africa, De Beer (2005) studied the dynamics of knowledge diffusion with specific reference to open access scholarly communication. She argued that knowledge diffusion and generation are the bases of sustainable economic growth by indicating that open access would go a long way in preventing marginalisation of science in and of developing countries, which, in turn, would reduce the levels of so-called "knowledge imperialism and dependence". In our context, this can easily be related to the reduction in restrictions for knowledge spillovers in the $R \& D$ sector of a developing economy.

5 As rightly indicated by one of the referees, the use of $R \& D$ requires adequate supplies of human capital, else the activity in this sector cannot be productive. Given this, one would probably want to broadly interpret the capital stock as incorporating both physical and human capital aspects. In any case, an increase in the degree of openness would improve the elasticity of knowledge creation with respect to the existing stock of knowledge and also affect the growth rate of the capital stock, besides the growth rate of new technology, which would, in turn, help propagate the process of technological progress further.

6 See below for further details in equation (17).

7 It is easy to see from Figure 3 that if $\beta+\theta=1$ there is no equilibrium. However, if along with $\beta+\theta=1, n=0$, we have infinitely many stable steady-state equilibria.

\section{References}

AGION, P. \& HOWITT, P. 1996. Research and development in the growth process. Journal of Economic Growth, 1:49-73.

BRANSTETTER, L. 1996. Are knowledge spillovers international or intranational in scope? Microeconometric evidence from the U.S. and Japan, NBER Working Paper No. 5800.

CHEN, PEI-PEI \& GUPTA, R. 2009. An investigation of openness and economic growth using panel estimation. Indian Journal of Economics. Forthcoming. COE, D.T. \& HELPMAN, E. 1995. International R\&D spillovers. European Economic Review, 39(5): 859-887. DE BEER, J.A. 2005. Open access scholarly communication in South Africa: A role for national information policy in the national system of innovation. Master of Philosophy (Information and Knowledge Management), University of Stellenbosch.

KELLER, W. 1998. Are international R\&D spillovers trade-related? Analyzing spillovers among randomly matched trade partners. European Economic Review, 42(8): 1469-1481.

NADIRI, M.I. \& KIM, S. 1996. International R\&D spillovers, trade and productivity in major OECD countries. NBER Working Paper No. W5801.

RODRIGUEZ, F. \& RODRIK, D. 2000. Trade policy and economic growth: A skeptic's guide to the crossnational evidence. NBER Macroeconomics Annual, 15: 261-325.

ROMER, D. 2006. Advanced microeconomics. ( $3^{\text {rd }}$ ed.) McGraw-Hill Companies Inc. NY. 